\title{
DEZA: auf die Bevölkerung ausgerichteter Ansatz
}

\section{Gerolf Weigel}

\section{(2) OpenEdition \\ 1 Journals}

Electronic version

URL: http://journals.openedition.org/sjep/551

DOI: $10.4000 /$ sjep.551

ISSN: 1663-9677

Publisher

Institut de hautes études internationales et du développement

\section{Printed version}

Date of publication: 1 novembre 2003

Number of pages: 139-143

ISSN: 1660-5926

\section{Electronic reference}

Gerolf Weigel, «DEZA: auf die Bevölkerung ausgerichteter Ansatz », Schweizerisches Jahrbuch für Entwicklungspolitik [Online], 22-2 | 2003, Online erschienen am: 10 Juni 2010, abgerufen am 08 September 2020. URL : http://journals.openedition.org/sjep/551 ; DOI : https://doi.org/10.4000/sjep. 551 


\section{DEZA: auf die Bevölkerung ausgerichteter Ansatz}

Gerolf Weigel*

D ie Informations- und Kommunikationstechnologien (IKT) verändern viele Facetten des menschlichen Lebens grundlegend. Dies gilt nicht nur für die Lern-, Arbeits- und Kommunikationsmethoden, sondern auch für die Interaktion zwischen Regierungen und Zivilgesellschaft. Die digitale Revolution ist eine stille Revolution, welche die im Werden begriffene Wissensgesellschaft nach und nach gestaltet.

Während die IKT denjenigen, die Zugang dazu haben, neue Möglichkeiten eröffnen, sind die anderen davon ausgeschlossen. Der digitale Graben ist der Ausdruck tiefgreifender sozialer, wirtschaftlicher und politischer Ungleichheiten. Eine engstirnige und „technizistische“ Vision des digitalen Grabens läuft Gefahr, kontraproduktive Aktionen hervorzurufen, die zu weiteren Ungleichheiten führen können. Die Frage der Herstellung von Inhalten ist zentral, ganz besonders von lokalen Inhalten, die der Entwicklung dienlich sind.

Nutzungen der IKT für die Entwicklung zu schaffen, ist eine neue Herausforderung, die eine Hoffnung für die marginalisierten Regionen der Erde darstellt, welche sich beteiligen und von den Möglichkeiten der globalen Wissensökonomie profitieren wollen.

Richtig eingesetzt, sind die IKT wirkungsvolle Instrumente für Emanzipation, Armutsbekämpfung und nachhaltige Entwicklung. Der Zugang zu den IKT kann in der Tat viele Vorteile mit sich bringen, namentlich indem er den Zugang zu relevanten Informationen erlaubt und denjenigen, denen es daran mangelt, Ausdrucksmöglichkeiten eröffnet. Dadurch können sich die Betreffenden besser Gehör verschaffen, ihre Bedürfnisse vortragen und die öffentliche Meinung warnen. Die IKT erleichtern ausserdem die gemeinschaftliche Bereitstellung und Nutzung von Kenntnissen und eröffnen neue Aussichten in Bezug auf die Möglichkeiten des Austausches zwischen Netzwerken und Institutionen.

Die Direktion für Entwicklung und Zusammenarbeit (DEZA) als führende Stelle in der Schweizer Regierung im Bereich „Informations- und Kommunika-

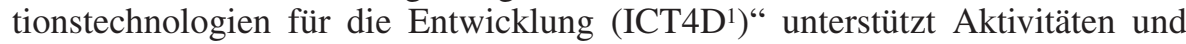
Projekte hauptsächlich durch Partnerschaften zwischen mehreren Akteuren (d.h. öffentlichen, privaten und gemeinnützigen Institutionen), und durch multilaterale Initiativen. Ausserdem weisen immer mehr Projekte der DEZA eine IKTKomponente auf. Der Schwerpunkt wird auf Inhalte und Wissensaufbau gelegt, was weit über die Informationsverbreitung im engeren Sinne hinausgeht.

DEZA, Abteilung ICT4D (Information and Communication Technologies for Development).

1 ICT4D: Information and Communication Technologies for Development. 
Mehrere schweizerische Nichtregierungsorganisationen und Medien sind Partner der DEZA in diesem Bereich. Der Weltgipfel über die Informationsgesellschaft (World Summit on the Information Society, WSIS) bietet die Gelegenheit, die Partnerschaften der DEZA auf schweizerischer Ebene, insbesondere durch die Schaffung einer NGO-Plattform sowie durch die Vertretung der Medien und der NGO in der offiziellen Schweizer Delegation, zu stärken.

\section{IKT und Entwicklung: der DEZA-Ansatz}

Das „ICT4D“-Konzept wurde vor kurzem von der internationalen Zusammenarbeit angenommen. Es bringt bereits verschiedene Politiken hervor, die sich schematisch in zwei Kategorien zusammenfassen lassen:

- Der auf die Technologien konzentrierte ,technizistische“ Ansatz ist am weitesten verbreitet, namentlich bei der Industrie, der Internationalen Fernmeldeunion (ITU) und bei einigen Programmen der Weltbank. Er beruht auf der Annahme, dass die Schaffung von Infrastruktureinrichtungen und Zugängen zu den IKT (z. B. durch den Anschluss von Schulen) automatisch Entwicklung hervorbringt. Der Ansatz konzentriert sich auf quantitative Ziele (,,Teledichte“, Durchlassbreite, usw.) und zielt darauf ab, den Ärmsten Zugang zum weltweiten Netz zu verschaffen, obwohl dieses durch einen asymmetrischen Informationsfluss vom Norden (hauptsächlich den Vereinigten Staaten und Europa) in den Süden gekennzeichnet ist. Dieser Ansatz beruht im Wesentlichen auf dem Prinzip des trickle down effect, auf Strategien zur Förderung von privaten Investitionen sowie auf der Einführung der IKT in die öffentlichen Verwaltungen und Sozialdienste.

- Der auf die „Entwicklung“ fokussierte Ansatz sieht die IKT seinerseits als machtvolle Instrumente, aber keinesfalls als Selbstzweck an. Er anerkennt, dass diese Technologien eine entscheidende Rolle spielen können, vorausgesetzt, dass sie in eine umfassendere Politik eingegliedert werden: in der Tat sind Kommunikation und Wissensaufbau komplexe Prozesse, die in einem sozioökonomischen Umfeld verankert sind, das jedes Mal einzigartig ist. Dieser Ansatz anerkennt die Wichtigkeit des Zugangs zur Weltinformation, misst aber der Schaffung und Verbreitung lokaler Inhalte ebensoviel Bedeutung bei. Die Entwicklungsländer müssen in der Lage sein, Inhalte zu schaffen, die auf ihren sozialen Werten beruhen, und die kulturelle Ausdrucksvielfalt wiedergeben. Der Ansatz beruht somit auf lokalen Prioritäten und auf lokaler Nachfrage. Er ergänzt die kommerziellen Ansätze und ermöglicht es, die benachteiligten Regionen und Bevölkerungsgruppen an die weltweite Infrastruktur der Telekommunikationen anzuschliessen.

Die DEZA folgt klar diesem zweiten Ansatz.

\section{Die IKT als Instrumente zur Armutsbekämpfung}

Die Informations- und Kommunikationstechnologien beschränken sich nicht auf das Internet und das Mobiltelefon, wenn auch diese beiden Instrumente die sichtbarsten und spektakulärsten sind. Die benachteiligten Bevölkerungsgrup- 
pen haben vorwiegend Zugang zu Radio und Fernsehen. Die Kombination der neuen Kommunikationsmittel mit den traditionellen Medien bietet ein grosses Potenzial, um so mehr als die herkömmlichen Medien, wie das Radio, immer interaktiver werden.

Wenn die IKT gut in Entwicklungsprogramme integriert sind, können sie Instrumente zur Armutsbekämpfung sein. Ein lokaler Radiosender kann zum Beispiel Informationen über Gesundheit oder Erziehung verbreiten, oder auch dazu beitragen, bei politischen Beschlussfassungen mehr Transparenz einzuführen.

Die Infrastruktureinrichtungen genügen jedoch nicht, und die IKT bergen auch die Gefahr von Rückschritten, insbesondere wenn die Mittel der internationalen Zusammenarbeit in allzu technische Projekte fliessen, die keine positiven Auswirkungen für die Ärmsten haben.

Die DEZA ist davon überzeugt, dass die IKT wirksame Instrumente zur Armutsbekämpfung darstellen. Die drei hauptsächlichen Stossrichtungen sind folgende :

$\square$ Zugang zu Information und Wissen. Der Zugang hängt vom Bestehen von Infrastruktureinrichtungen und von menschlichen und institutionellen Fähigkeiten zur Nutzung der Informationen ab. Er hängt auch von den wirtschaftlichen Fähigkeiten der Bevölkerung ab, den Informationszugang bezahlen zu können. Dabei handelt es sich nicht unbedingt um individuelle Zugänge. Gemeinschaftszugänge sind, vom wirtschaftlichen, sozialen und kulturellen Standpunkt aus gesehen, oftmals angebrachter. Es geht darum, diese Option vermehrt zu entwickeln.

$\square$ Ausdrucksmöglichkeiten und Selbsthilfe. Die IKT können die Ausdrucksmöglichkeiten der Staatsbürger erleichtern, insbesondere um die Entwicklungspolitik $\mathrm{zu}$ orientieren und dezentralisiertere Beschlussverfahren $\mathrm{zu}$ schaffen. Die IKT erlauben es auch, die soziokulturelle Vielfalt zu fördern, namentlich indem sie allen Bürgern gleichen Zugang zu den Quellen des Wissens, zur Information und zu den Medien geben.

$\square$ Kommunikation, Netzwerke und Austausch. Der Wissensaustausch ist besonders wegen seiner dezentralisierten Funktionsweise und aufgrund des Tempos der sich ergebenden Veränderungen - in der Informationsgesellschaft von entscheidender Bedeutung. Die IKT erleichtern den Aufbau von Partnerschaften, Koalitionen und neuen Bündnissen. Die interkulturelle Kommunikation wird auch durch die Intensivierung des Austausches zwischen verschiedenen Kulturen erleichtert.

\section{Die Programme der DEZA}

Wie gesagt hat die DEZA in den schweizerischen Regierungsinstanzen die führende Rolle auf dem Gebiet der Informations- und Kommunikationstechnologien für die Entwicklung inne. Ihre Abteilung „ICT4D“ koordiniert die Programme. Das Staatssekretariat für Wirtschaft (seco) unterstützt seinerseits Projekte im Bereich des elektronischen Handels. Das Bundesamt für Kommunikation (BAKOM) arbeitet, als Mitglied der Internationalen Fernmeldeunion 
(ITU) und als führendes Amt für die Schweiz bei der Organisation des Weltgipfels zur Informationsgesellschaft (WSIS), auf diesem Gebiet mit der DEZA zusammen.

Die DEZA war immer der Ansicht, dass Wissen das wichtigste Kapital für die Entwicklung ist; dies gilt jetzt um so mehr mit der Einführung der digitalen Technologien. Unter Berücksichtigung ihres Entwicklungsansatzes fördert die DEZA weder grosse Infrastrukturprojekte, noch traditionelle bilaterale Projekte. Sie stützt sich auf existierende Netzwerke, um die institutionellen und organisatorischen Grundlagen zu stärken und die Ausdrucksmöglichkeiten der Länder des Südens - vor allem der benachteiligten Bevölkerungsgruppen - in den weltweiten und regionalen Netzwerken zu erleichtern, in der Hoffnung, dass sie dadurch vermehrt an den Beschlussfassungen teilnehmen können.

Es geht auch darum, die lokalen Organisationen und Netzwerke zu stärken, um den Süd-Süd-Dialog und den Wissensaustausch zu erleichtern. Die Förderung, Anerkennung, Verwendung und der Austausch von lokalem Wissen sind ebenfalls eine der Prioritäten der DEZA. Ihr Einsatz besteht hauptsächlich darin, multilaterale Initiativen und Partnerschaften zwischen mehreren Akteuren (multistakeholder partnerships) zu unterstützen. In der Tat liegt die Hauptstärke der IKT darin, die Errichtung von Netzwerken und Partnerschaften zwischen verschiedenen institutionellen Kreisen - von den Organisationen vor Ort über die Privatunternehmen bis hin zu den Regierungsinstanzen - zu ermöglichen.

\section{Lehren und Ausrichtungen}

Die Informations- und Kommunikationstechnologien sind in den Entwicklungsländern bereits zur Realität geworden. Sie schaffen effektiv auch neue Ungleichheiten, nämlich zwischen denjenigen, die Zugang zu den IKT haben, und denjenigen, die keinen Zugang haben. Deshalb besteht bei den Entwicklungsländern eine starke Nachfrage nach Projekten mit einem Entwicklungsansatz. Die Hilfsorganisationen dürfen diesen Appell nicht ignorieren. Sie müssen ihre Programme entsprechend ausrichten, um die IKT zu Werkzeugen der Armutsbekämpfung zu machen².

Um diese Ausrichtung wirksam zu gestalten, ist es wesentlich, parallel dazu eine Kultur des Erfahrungsaustausches zu schaffen, um besser Lehren aus den Erfolgen und Fehlschlägen der verschiedenen Initiativen im ICT4D-Bereich ziehen zu können. Es geht darum, die Erfolgskriterien für die Herstellung von Inhalten und für den Ausbau der institutionellen und menschlichen Fähigkeiten eingehend zu prüfen. Es geht ebenfalls darum, über Fragen in Bezug auf die Kosten und auf lebensfähige Wirtschaftsmodelle für die Ärmsten nachzudenken.

2 Gerster R. \& Zimmermann S., ICT for Poverty Reduction? SDC Discussion Paper, Bern, März 2003. 


\section{Wichtigste Programme und Partner*}

\begin{tabular}{lll}
\hline Programme/Partner & Beteiligung der DEZA & Internet-Adressen \\
\hline The Global Knowledge & Der Direktor der DEZA ist & <www.globalknowledge.org $>$ \\
Partnership (GPK) & Vorsitzender des GPK-Exekutiv- & \\
(Partnerschaft zwischen öffent- & komitees. Die DEZA beteiligte sich & \\
$\begin{array}{l}\text { lichen, privaten und gemein- } \\
\text { nützigen Organisationen) }\end{array}$ & $\begin{array}{l}\text { intensiv an der Erarbeitung der } \\
\end{array}$ & $\begin{array}{l}\text { GPK-Strategie 2005 und der Ein- } \\
\text { richtung eines ständigen GPK- } \\
\end{array}$ \\
& Sekretariats in Kuala Lumpur.
\end{tabular}

Weltgipfel über die Informationsgesellschaft (WSIS), ICT4D-Plattform und World Die DEZA organisiert eine Plattform <www.ict-4d.org > für "multi-stakeholder"-Debatten und -Ausstellungen zu Fragen im Electronic Media Forum Zusammenhang mit den IKT und (WEMF) der Entwicklung. Sie finanziert auch das WEMF mit.

International Institute for Communications and Development (IICD)

Building Digital Opportunities (BD0)
Die DEZA kofinanziert Projekte, Aktivitäten und Anlässe, insbesondere in Mali und Bolivien.
$<$ www.iicd.org >

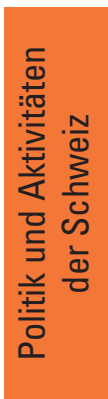

\section{One World International (OWI) Die DEZA unterstützt die südasiati- <www.oneworld.net> sche Plattform dieses weltweiten Netzes für die Entwicklung.}

UN ICT Task Force

Der Direktor der DEZA ist Mitglied $<$ www.unicttaskforce.org> des Beratungsausschusses. Die DEZA lässt dieser von der UNO lancierten globalen Plattform auch finanzielle Unterstützung zukommen.

Schweizerische Fachstelle für Informationstechnologien im Bildungswesen (SFIB)
Die DEZA ist Partnerin der SFIB, um <www.educa.ch> das elektronische Lernen und den internationalen Informationsaustausch zwischen Schulen zu fördern.

Verschiedene Partnerschaften Die DEZA finanziert andere Anlässe und Projekte mit, insbesondere diejenigen der UN-Wirtschaftskommission für Afrika (ECA), <www.uneca.org > ; der AMARC (World Association of Community Radio Broadcasters), <www.amarc.org/africa>; von Brot für alle, <www.ppp.ch> und von CRIS Bolivien.

* Vollständige Liste der Programme und Partner unter : <www.sdc.admin.ch/ict4d>. 